\title{
The far-infrared/radio correlation in the ISO era ${ }^{\star \star \star}$
}

\section{The warm and cold far-infrared/radio correlations}

\author{
D. Pierini ${ }^{1}$, C. C. Popescu ${ }^{2,3}$, R. J. Tuffs ${ }^{2}$, and H. J. Völk ${ }^{2}$ \\ 1 Max-Planck-Institut für extraterrestrische Physik, Giessenbachstr., 85748 Garching, Germany \\ 2 Max-Planck-Institut für Kernphysik, Saupfercheckweg 1, 69117 Heidelberg, Germany \\ e-mail: [Cristina.Popescu;Richard.Tuffs; Heinrich.Voelk] @mpi-hd.mpg.de \\ 3 Research Associate, The Astronomical Institute of the Romanian Academy, Str. Cuţitul de Argint 5, Bucharest, Romania
}

Received 19 March 2003 / Accepted 29 July 2003

\begin{abstract}
We present the correlation between the far-infrared (FIR) and radio emissions from a composite sample of 72 nearby normal galaxies observed with the ISOPHOT instrument on board the Infrared Space Observatory. The galaxies in the sample have measurements at three FIR wavelengths $(60,100$ and $170 \mu \mathrm{m})$, which allowed a direct determination of the warm and cold FIR emission components. This is the first time that the correlation has been established for the total FIR luminosity, of which most is carried by the cold dust component predominantly emitting longwards of the spectral coverage of IRAS. The slope of this correlation is slightly non-linear $(1.10 \pm 0.03)$. Separate correlations between the warm and cold FIR emission components and the radio emission have also been derived. The slope of the warm FIR/radio correlation was found to be linear (1.03 \pm 0.03$)$. For the cold FIR/radio correlation we found a slightly non-linear $(1.13 \pm 0.04)$ slope. We qualitatively interpret the correlations in terms of star formation rate and find that both the FIR and radio emissions may be consistent with a non-linear dependence on star formation rate for galaxies not undergoing starburst activity.
\end{abstract}

Key words. galaxies: spiral - galaxies: stellar content - galaxies: ISM - infrared: galaxies - radio continuum: galaxies

\section{Introduction}

One of the most surprising discoveries of the IRAS all-sky survey was the very tight and universal correlation between the spatially integrated far-infrared (FIR) and radio continuum emissions of late-type galaxies (de Jong et al. 1985; Helou et al. 1985; Wunderlich et al. 1987). The standard interpretation of the so-called FIR/radio correlation is in terms of massive star formation activity (see Condon 1992; Völk \& Xu 1994 for reviews). This picture assumes that the FIR emission is due to dust heated by the massive stars which are also responsible for the radio emission: the ionising radiation from the massive stars powers the thermal radio emission, and the remnants of supernova explosions which occur at the end of their lives presumably accelerate the cosmic ray electrons. The link between the radio synchrotron and dust emissions was quantified

Send offprint requests to: D. Pierini, e-mail: dpierini @mpe.mpg. de * Based on observations with the Infrared Space Observatory (ISO), an ESA project with instruments funded by ESA member States (especially the PI countries: France, Germany, The Netherlands, and the UK) and with the participation of ISAS and NASA.

$\star \star$ Table 2 and Appendices A and B are only available in electronic form at http://www. edpsciences.org by the calorimeter theory of Völk (1989) (see also Lisenfeld et al. 1996a). Specific aspects of the FIR/radio correlation have been addressed by many authors (e.g. Gavazzi et al. 1986; Cox et al. 1988; Devereux \& Eales 1989; Chi \& Wolfendale 1990; Condon et al. 1991; Price \& Duric 1992; Xu et al. 1994a; Xu et al. 1994b; Niklas et al. 1995; Lisenfeld et al. 1996b; Bressan et al. 2002; Bell 2003; Groves et al. 2003).

All these studies of the FIR/radio correlation were based on IRAS measurements and FIR luminosities obtained by using the IRAS flux densities at 60 and $100 \mu \mathrm{m}$. However the FIR spectral energy distribution (SED) of normal ${ }^{1}$ galaxies typically peaks longwards of $100 \mu \mathrm{m}$, beyond the spectral grasp of IRAS, as predicted from submillimeter (submm) observations by Chini et al. (1986). With the advent of the Infrared Space Observatory (ISO) (Kessler et al. 1996), in particular of the ISOPHOT instrument (Lemke et al. 1996) on board ISO, it became routinely possible to measure the peak of the FIR emission, also for fainter objects not detected by IRAS. It was found that, especially for quiescent galaxies, most of the FIR luminosity is carried by a cold dust component (Popescu et al. 2002) whose luminosity is not well predicted by the

\footnotetext{
${ }^{1}$ By "normal" we loosely refer to galaxies which are not dominated by an active nucleus and whose current star formation rates would be sustainable for a substantial fraction of a Hubble time.
} 
IRAS measurements. In fact the luminosity correction factors ${ }^{2}$ for cold dust do not correlate with Hubble type and exhibit a huge scatter, with mean values ranging from 1 to 3 (Popescu $\&$ Tuffs 2002b). In view of this, there is a clear need to redefine the FIR/radio correlation in terms of the total FIR-submm luminosity, as derived from ISO measurements.

Another result to emerge from ISO is the confirmation that the FIR SEDs of normal galaxies require a warm and a cold dust emission component to be fitted (for a review see Tuffs \& Popescu 2003). Although the concept of warm and cold emission components is as old as IRAS (de Jong et al. 1984), it only became possible to directly measure and separate these components with ISO, due to its spectral grasp and multi filter coverage of the FIR regime. The warm component can be identified with locally heated dust in HII regions ${ }^{3}$ and the cold component with dust distributed in the general diffuse interstellar medium and heated by a combination of non-ionising ultraviolet (UV) and optical/near-IR photons. This interpretation is consistent with what has been seen in the ISOPHOT maps of nearby galaxies (e.g. Haas et al 1998; Hippelein et al. 2003). It is also consistent with models of the UV/optical/FIR/submm SEDs in normal galaxies, which self-consistently calculate a continuous distribution of dust temperatures based on radiativetransfer calculations (Silva et al. 1998; Popescu et al. 2000; Misiriotis et al. 2001). Thus ISO measurements give us the first direct opportunity of defining the FIR/radio correlation separately for the warm and cold dust components. In the past this could only be indirectly attempted by constraining the warm dust component to be proportional to the $\mathrm{H} \alpha$ emission, as done by Xu et al. (1994b). These authors established separate relations for the warm FIR/radio correlation and the cold FIR/radio correlation. However, the cold FIR luminosity derived in this study was based on IRAS data, and misses the bulk of the true cold dust luminosity. Other authors have used different operational recipes with different physical interpretations to derive warm and cold FIR emission components from IRAS data for comparison with radio data (e.g. Beck \& Golla 1988; Fitt et al. 1988; Wunderlich \& Klein 1991; Condon et al. 1991; Hoernes et al. 1998), but again these studies missed the bulk of the cold dust emission.

In this paper we redefine the FIR/radio correlation using for the first time the total FIR luminosity (as measured with ISO) for a statistical sample. Plots of the FIR/radio correlation using the new ISO measurements were also given in Popescu et al. (2002), but only for a very small sample, and therefore no attempt was made to quantify the linearity of the correlation there. The goal of this paper is to provide a statistical analysis of the correlation and also to derive the slope of the correlation separately for the warm and cold dust components. Finally the

\footnotetext{
2 These correction factors are the multiplicative factors by which the total FIR luminosity (40-1000 $\mu \mathrm{m})$, as derived from ISO measurements, differs from the IRAS FIR luminosity, derived from the formula (Helou et al. 1988): $f_{40-120}\left[\mathrm{~W} \mathrm{~m}^{-2}\right]=1.26 \times 10^{-14}\left(2.58 f_{60 \mu \mathrm{m}}+\right.$ $f_{100 \mu \mathrm{m}}$ ), with $f_{60 \mu \mathrm{m}}$ and $f_{100 \mu \mathrm{m}}$ expressed in Jy.

${ }^{3}$ In the context of dust emission we use the term HII regions to denote not only the optical emitting HII regions within the star forming complexes, but also their immediate vicinity where grains can also be strongly heated by the massive stars.
}

new results are discussed in the context of predictions of theoretical models on photon heating of dust in normal star-forming galaxies.

The paper is organised as follows. In Sect. 2 we describe the sample selection and the analysis of the radio data. In Sect. 3 we present the results for the total FIR/radio correlation, as well as for the warm and cold FIR/radio correlations. Each of these correlations is qualitatively analysed in terms of its dependence on the star formation rate in Sect. 4. In Sect. 5 we discuss scenarios which can simultaneously account for both the warm and cold FIR/radio correlations. A summary is given in Sect. 6.

\section{The ISO sample: Selection and data analysis}

The sample used in this study is a combination of a very deep, optically selected sample of normal, relatively quiescent galaxies together with a shallow, FIR selected sample of relatively bright galaxies. This provides us with a high dynamic range in FIR luminosities as well as good statistics. For this purpose we used the ISOPHOT Virgo Cluster Deep Survey (Tuffs et al. 2002a,b), which is the deepest survey (both in luminosity and surface brightness terms) of normal galaxies performed by ISOPHOT, and the ISOPHOT $170 \mu \mathrm{m}$ Serendipity Survey (Stickel et al. 2000). All galaxies in the combined sample have had their integrated flux densities measured by ISOPHOT at $170 \mu \mathrm{m}$ (the Virgo galaxies also in the ISOPHOT bands centered at 60 and $100 \mu \mathrm{m}$ ). Most galaxies from the Virgo sample are freshly falling in from the field (Tully \& Shaya 1984; Tuffs et al. 2002a,b) and therefore we consider it legitimate to combine it with the ISOPHOT $170 \mu \mathrm{m}$ Serendipity Survey sample. Furthermore there is no evidence for an effect of the cluster environment on the FIR properties of the galaxies from the ISOPHOT Virgo Cluster Deep Sample (Popescu et al. 2002; Popescu \& Tuffs 2002b). This is to be expected since the bulk of the FIR emission arises primarily from the inner regions of the galactic disks. The radio emission may be more prone to alterations due to environmental effects, as we will discuss in Sect. 3. However we will show there that, in the case of our sample, these effects are only of second order. Thus, in this paper we regard the main distinction between Virgo and Serendipity Survey galaxies to be the quiescent nature of the former, rather than their association with the cluster environment. So, put together, the combined sample will provide us with larger statistics and a good dynamic range in star formation activity.

In total 72 galaxies were selected, as described below. Of these, 20 galaxies show some evidence for LINER/Seyfert activity or are pair/multiple/merger systems.

\subsection{The VIRGO subsample}

The ISOPHOT Virgo Cluster Deep Sample consists of 63 galaxies later than $\mathrm{S} 0$ and brighter than $B_{\mathrm{T}}=16.8$, selected from the Virgo Cluster Catalogue (VCC) of Binggeli et al. (1985). Tuffs et al. (2002a,b) have presented deep diffraction-limited FIR strip maps of this sample, obtained with 
ISOPHOT at the central wavelengths 60,100 and $170 \mu \mathrm{m}^{4}$. Details of observations, data reduction, estimate of the calibration uncertainty and procedure of extraction of photometry are given in Tuffs et al. (2002a,b). As discussed by these authors, the 63 galaxies represent a sample of nearby normal (i.e. non-AGN dominated and non-starburst) late-type galaxies. Nevertheless a few objects have also some mild LINER/Seyfert activity.

Out of this sample, 38 galaxies (i.e. 63.5 per cent of the total) were detected at all three wavelengths. Most of them were discovered to contain a cold dust emission component which could not have been recognised by IRAS, irrespective of the morphological classification of individual objects (Popescu et al. 2002). The ISOPHOT FIR SEDs of these galaxies were fitted with a combination of two modified blackbody functions, physically identified with the emissions from a localised warm dust component, with a fixed temperature of $47 \mathrm{~K}$, and a diffuse cold dust component (see Popescu et al.). The cold dust temperatures were found to span a broad range, with a median of $18 \mathrm{~K}$. The physical justification for fixing the temperature of the warm component is based on the modelling work of Popescu et al. (2000) and Misiriotis et al. (2001). The latter papers showed that $\sim 20$ per cent of the integrated FIR emission at $60 \mu \mathrm{m}$ comes from stochastically heated grains in the diffuse interstellar medium (ISM), $\sim 20$ per cent from optically heated grains in the diffuse ISM, and $\sim 60$ per cent from HII regions. So it is a good physical approximation to consider that most of the emission at $60 \mu \mathrm{m}$ comes from grains within the HII regions (which do not emit stochastically). Although there is a variation in grain temperature between HII regions in any given galaxy (Peeters et al. 2002) (due to changes in size, geometry and exciting stars), these are quite moderate (in comparison to the systematic difference in temperature between grains in HII regions and grains in the diffuse ISM). Thus, if we average over the ensemble of HII regions in each galaxy we would expect the ensemble of HII regions to have virtually identical SEDs from one galaxy to the next.

Fourteen of the 38 galaxies with both warm and cold dust emission components have also been detected in the radio continuum at $1.4 \mathrm{GHz}$, thanks to the recent NRAO/VLA Sky Survey (NVSS) (Condon et al. 1998). For these objects we performed aperture photometry to extract integrated flux densities from the NVSS maps (see Appendix A, only available in the on-line edition of the journal). The 14 VCC galaxies with FIR and radio counterparts are considered in the present study. They constitute the so-called "VIRGO" subsample.

Table 1 reproduces the parameters relevant to this investigation for the individual VIRGO objects as follows:

Column 1: VCC denomination.

Column 2: morphological type (from the VCC, as in Tuffs et al. 2002a,b).

Column 3: total radio continuum luminosity at $1.4 \mathrm{GHz}$ $\left(L_{1.4 \mathrm{GHz}}\right)$ with its error (see Appendix A, only available in the

\footnotetext{
${ }^{4}$ A subsample of the ISOPHOT Virgo Cluster Deep Sample has been also observed with the Long Wavelength Spectrometer at the $158 \mu \mathrm{m}$ [CII] fine-structure gas cooling line (Leech et al. 1999) and analysed by Pierini and collaborators (1999, 2001, 2003).
}

on-line edition of the journal), in decimal logarithmic units.

Column 4: total FIR continuum luminosity $\left(L_{\mathrm{FIR}}\right)$, in decimal logarithmic units. The uncertainty in this luminosity is $\sim 15$ per cent (random) for all the sample objects.

Columns 5, 6: FIR continuum luminosities from the warm and cold dust emission components ( $L_{\mathrm{FIR}}^{\mathrm{warm}}$ and $L_{\mathrm{FIR}}^{\text {cold }}$ ), respectively (from Popescu et al. 2002), in decimal logarithmic units. We adopt a conservative value of 20 per cent (random) for the uncertainty of each of these luminosities for all the sample objects.

Column 7: $L_{40-120}$, i.e., the FIR luminosity that IRAS would have derived based on the Helou et al. (1988) formula: $f_{40-120}\left[\mathrm{~W} \mathrm{~m}^{-2}\right]=1.26 \times 10^{-14}\left(2.58 f_{60 \mu \mathrm{m}}+f_{100 \mu \mathrm{m}}\right)$, where $f_{60 \mu \mathrm{m}}$ and $f_{100 \mu \mathrm{m}}$ are expressed in Jy. The luminosity is given in decimal logarithmic units. We adopt a conservative value of 30 per cent for the uncertainty of this luminosity for all sample objects.

Column 8: individual notes.

Luminosities are determined by assuming that all the galaxies have the same distance of $11.5 \mathrm{~h}^{-1} \mathrm{Mpc}$ (Binggeli et al. 1993), where $h$ is the Hubble constant in units of $100 \mathrm{~km} \mathrm{~s}^{-1} \mathrm{Mpc}^{-1}$.

\subsection{The ISOPHOT Serendipity Survey subsample}

A sample of 115 nearby galaxies was presented by Stickel et al. (2000) as a first result of the "ISOPHOT $170 \mu \mathrm{m}$ Serendipity Survey" (ISS). We refer the reader to these authors for details about observations, data reduction, estimate of the calibration uncertainty, procedure of extraction of photometry and optical/FIR identification.

As recognised by Stickel et al., several of their objects have Seyfert/LINER activity and/or are pair/multiple/merging systems.

Not all galaxies from the ISS catalogue could be used in the present study. Out of the 115 ISS galaxies we first rejected 3 not having IRAS detections at both 60 and $100 \mu \mathrm{m}$ and 13 galaxies which either have no morphological information or are classified as S0, elliptical or peculiar. Of the remaining 99 galaxies we omitted 3 already included in the VIRGO subsample. Furthermore, 26 galaxies of the remaining $96 \mathrm{did}$ not have a radio counterpart or were confused in the NVSS at $1.4 \mathrm{GHz}$. Radio counterparts are associated with optically catalogued galaxies when the distance of the peak radio surface brightness of the potential radio identification from the optical position of the target galaxy is within the NVSS resolution. Furthermore, one galaxy was dropped because it has an inclination greater than $\sim 70^{\circ}$ and an apparent major axis longer than $\sim 4^{\prime}$. As discussed in Appendix B (only available in the on-line edition of the journal), such galaxies may have flux densities overestimated by a factor of 2 . Finally, only galaxies with heliocentric velocities higher than $1500 \mathrm{~km} \mathrm{~s}^{-1}$ are selected. The reason is as follows. For most of the galaxies in the ISS catalogue only kinematical distances are available from individual redshifts, as listed by Stickel et al. (2000). The assumption of a free Hubble Flow does not apply to the relatively nearby galaxies. Hence 11 galaxies which are not members of 
Table 1. Galaxy parameters for the VIRGO subsample.

\begin{tabular}{|c|c|c|c|c|c|c|c|}
\hline $\begin{array}{l}\text { Den. } \\
\text { VCC }\end{array}$ & Hubble type & $\begin{array}{c}\log L_{1.4 \mathrm{GHz}} \\
{\left[\mathrm{W} \mathrm{Hz}^{-1} \mathrm{~h}^{-2}\right]}\end{array}$ & $\begin{array}{l}\log L_{\mathrm{FIR}} \\
{\left[\mathrm{W} \mathrm{h}^{-2}\right]}\end{array}$ & $\begin{array}{c}\log L_{\mathrm{FIR}}^{\mathrm{warm}} \\
{\left[\mathrm{W} \mathrm{h}^{-2}\right]}\end{array}$ & $\begin{array}{l}\log L_{\mathrm{FIR}}^{\text {cold }} \\
{\left[\mathrm{W} \mathrm{h}^{-2}\right]}\end{array}$ & $\begin{array}{c}\log L_{40-120} \\
{\left[\mathrm{~W} \mathrm{~h}^{-2}\right]}\end{array}$ & Notes \\
\hline 66 & $\mathrm{SBc}$ & $20.87 \pm 0.21$ & 35.71 & 35.22 & 35.53 & 35.36 & \\
\hline 92 & $\mathrm{Sb}:$ & $21.18 \pm 0.16$ & 36.19 & 35.52 & 36.09 & 35.67 & LINER $^{\mathrm{a}, \mathrm{b}}$ \\
\hline 152 & Scd & $20.53 \pm 0.23$ & 35.60 & 35.02 & 35.46 & 35.31 & \\
\hline 460 & Sa pec & $20.54 \pm 0.23$ & 35.78 & 35.21 & 35.64 & 35.55 & LINER $^{\mathrm{c}}$ \\
\hline 873 & $\mathrm{Sc}$ & $20.65 \pm 0.23$ & 35.90 & 35.41 & 35.74 & 35.57 & \\
\hline 971 & $\mathrm{Sd}$ & $19.97 \pm 0.12$ & 34.80 & 34.22 & 34.67 & 34.50 & \\
\hline 1002 & $\mathrm{SBc}$ & $20.06 \pm 0.15$ & 35.37 & 34.67 & 35.27 & 35.06 & \\
\hline 1043 & Sb(tides) & $21.08 \pm 0.18$ & 35.83 & 35.16 & 35.72 & 35.44 & LINER $^{\mathrm{d}}$ \\
\hline 1110 & Sab pec & $20.34 \pm 0.21$ & 35.63 & 35.08 & 35.48 & 35.24 & LINER $^{\mathrm{e}}$ \\
\hline 1379 & $\mathrm{SBc}$ & $20.10 \pm 0.16$ & 35.37 & 34.81 & 35.24 & 35.09 & \\
\hline 1554 & $\mathrm{Sm}$ & $21.33 \pm 0.13$ & 35.85 & 35.55 & 35.55 & 35.67 & \\
\hline 1575 & $\mathrm{SBm}$ pec & $19.96 \pm 0.12$ & 35.21 & 34.84 & 34.96 & 34.99 & \\
\hline 1690 & $\mathrm{Sab}$ & $21.13 \pm 0.17$ & 36.12 & 35.59 & 35.97 & 35.81 & LINER, Sy $y^{\mathrm{d}, \mathrm{f}, \mathrm{g}}$ \\
\hline 1727 & $\mathrm{Sab}$ & $21.26 \pm 0.15$ & 36.07 & 35.43 & 35.95 & 35.66 & LINER, Sy $1.9^{\mathrm{d}, \mathrm{f}}$ \\
\hline
\end{tabular}

${ }^{\text {a }}$ Rauscher (1995); ${ }^{\text {b }}$ Barth et al. (1998); ${ }^{\mathrm{c}}$ Ho et al. (1995); ${ }^{\mathrm{d}}$ Ho et al. (1997); ${ }^{\mathrm{e}}$ Gonzalez-Delgado et al. (1997); ${ }^{\mathrm{f}}$ Stauffer (1982); ${ }^{\mathrm{g}}$ Keel (1983).

the Virgo Cluster and have heliocentric velocities lower than $1500 \mathrm{~km} \mathrm{~s}^{-1}$ were dropped from the sample.

Hereafter we refer to the final 58 galaxies selected from the ISS catalogue as the ISS subsample. For all galaxies in the ISS subsample we determined new warm and cold dust temperatures from the composite IRAS and ISOPHOT data listed by Stickel et al. using the method described in Popescu et al. (2002). For this purpose we first applied ISO/IRAS corrections for the original IRAS flux densities at 60 and $100 \mu \mathrm{m}$ and ISO/DIRBE corrections for the ISOPHOT flux densities at $170 \mu \mathrm{m}$, both corrections being taken from Tuffs et al. (2002a,b). The SEDs of all these objects required both warm and cold dust emission components to be fitted.

We assigned distances to the members of the ISS subsample as follows. We identify 3 galaxies from the ISS subsample as members of the Virgo cluster (and not in the ISOPHOT Virgo Cluster Deep sample) and, thus, we assign them to be at the common distance of $11.5 \mathrm{~h}^{-1} \mathrm{Mpc}$ adopted for the VIRGO subsample (cf. Sect. 2.1). For the other galaxies of the ISS subsample we have no clue whether they belong to the field or to a group/cluster, though we know if they are mergers or not. We determine kinematical distances, corrected to the Galactic Standard of Rest with the NED Velocity Correction Calculator, independent of whether they are isolated or not and whether they are mergers or not.

Table 2, only available in the on-line edition of the journal, reproduces the parameters relevant to this investigation for the individual ISS objects as follows:

Column 1: galaxy denomination (from Stickel et al. 2000).

Column 2: morphological type (from NED).

Column 3: total radio continuum luminosity at $1.4 \mathrm{GHz}$ $\left(L_{1.4 \mathrm{GHz}}\right)$ with its error (see Appendix A, only available in the on-line edition of the journal), in decimal logarithmic units.

Column 4: total FIR continuum luminosity $\left(L_{\mathrm{FIR}}\right)$, in decimal logarithmic units. The uncertainty in this luminosity is $\sim 15$ per cent (random) for all the sample objects.
Columns 5, 6: FIR continuum luminosities from the warm and cold dust emission components ( $L_{\mathrm{FIR}}^{\mathrm{warm}}$ and $L_{\mathrm{FIR}}^{\text {cold }}$ ), respectively (this work), in decimal logarithmic units. We adopt a conservative value of 20 per cent (random) for the uncertainty of each of these luminosities for all the sample objects.

Column 7: the IRAS FIR luminosity $L_{40-120}$ (calculated as in Table 1), in decimal logarithmic units. We adopt a conservative value of 30 per cent for the uncertainty of this luminosity for all the sample objects.

Column 8: galaxy distance.

Column 9: individual notes.

\section{Results}

\subsection{The total FIR/radio correlation}

The relationship between the total (warm + cold) FIR emission, $L_{\mathrm{FIR}}$, and the radio continuum emission at $1.4 \mathrm{GHz}$ for the 72 sample galaxies is reproduced in Fig. 1. Here the solid line reproduces the linear fit to the data (in $\log -\log$ ) of equation:

$\log L_{1.4 \mathrm{GHz}}=m( \pm \epsilon(m)) \log L_{\mathrm{FIR}}+c( \pm \epsilon(c)) \mathrm{W} \mathrm{Hz}^{-1} \mathrm{~h}^{-2}$,

where the parameters of this fit are given in Table 3, together with its reduced $\chi^{2}$ and the dispersion around the fitted relation. In obtaining the fits we adopted the algorithm executing a bivariate least-squares fit, taking into account the uncertainties in both the $x$ - and $y$-variables at the same time, as implemented in the task "FITEXY" of the Numerical Recipes (Press et al. 1992).

Figure 1 confirms that there is a tight correlation between dust and radio continuum emission, extending over three orders of magnitude in both luminosities. The total FIR/radio correlation is only slightly non-linear, with a slope $m=1.10 \pm 0.03$. We note that a luminosity-luminosity correlation analysis will be biased towards linearity, because it includes also the scaling 


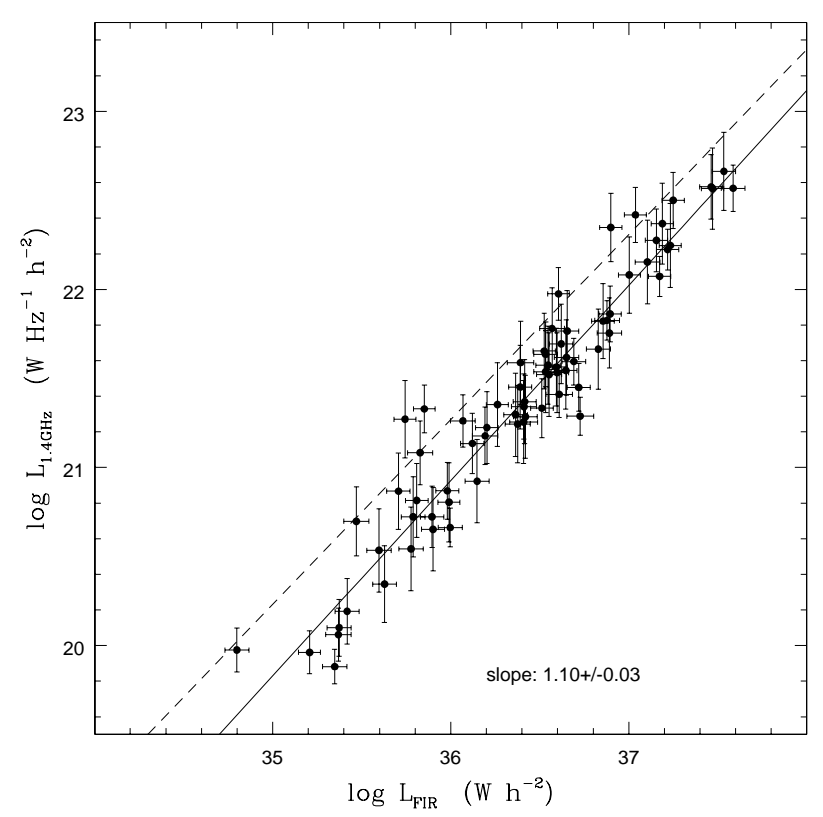

Fig. 1. The radio luminosity at $1.4 \mathrm{GHz}\left(L_{1.4 \mathrm{GHz}}\right)$ vs. the total FIR luminosity (of the warm + cold dust emission components) $\left(L_{\mathrm{FIR}}\right)$ for the full sample of 72 objects under investigation. The plot shows a tight correlation between $L_{1.4 \mathrm{GHz}}$ and $L_{\mathrm{FIR}}$, over three orders of magnitude in both luminosities. From the fit (solid line) we conclude that this correlation is only slightly non-linear (see the slope of the fit and its uncertainty, here indicated). The dashed line represents the fit to the $L_{1.4 \mathrm{GHz}}$ vs. $L_{40-120}$ correlation, which is the equivalent of what would have been derived from IRAS.

effect due to galaxy size (or mass). Therefore, it is to be expected that the derived slope is only a lower limit to the slope that would be obtained from an analysis of normalised luminosities (see Xu et al. 1994a).

As a comparison, in Fig. 1 we also plot the fit to the $L_{1.4 \mathrm{GHz}}$ vs. $L_{40-120}$ correlation, which is the equivalent of what would have been derived from IRAS. The statistics for this fit are also given in Table 3. There is an obvious shift of up to 0.4 in $\log L_{\text {FIR }}$, which is produced by the inclusion of the cold dust component, mainly not seen by IRAS. This shift is more pronounced at the faint end of the correlation, where the cold dust component is more dominant. This also leads to a slight increase in non-linearity when the total FIR luminosity is taken into account instead of $L_{40-120}$.

The fact that some galaxies show evidence for mild LINER/Seyfert activity or are in pair/multiple/merging systems does not seem to affect the shape of the correlation (not shown). In fact, we obtain $m=1.09 \pm 0.07$ for the subsample of 20 galaxies with some activity (Table 3 ) and $m=1.10 \pm 0.04$ for the remainder. Since accretion powered systems are known not to lie on the FIR/radio correlation (Sopp \& Alexander 1991), this implies that even for the objects in our sample with some evidence for AGN activity, the FIR emission is powered by star formation activity and not by accretion (cf. Rodriguez Espinosa et al. 1987).

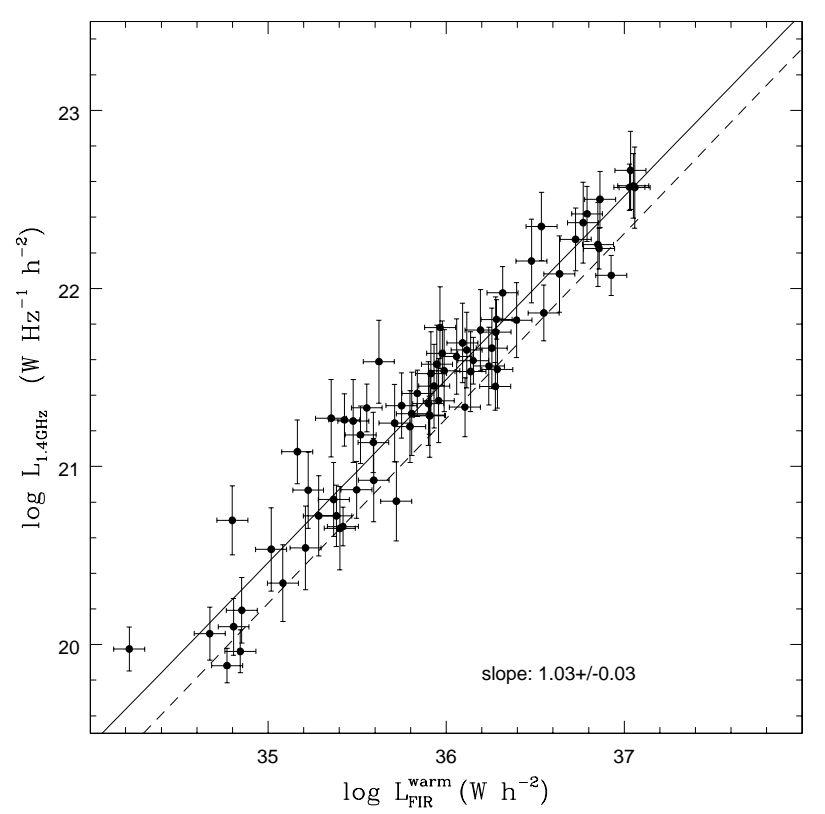

Fig. 2. The radio luminosity at $1.4 \mathrm{GHz}$ vs. the FIR luminosity of the warm component $\left(L_{\mathrm{FIR}}^{\mathrm{warm}}\right)$ for the full sample of 72 objects under investigation. The plot shows a tight correlation between these two emissions, over three orders of magnitude in both luminosities. The fit to the correlation (plotted with the solid line) indicates a linear relation. The value of the slope and its uncertainty are indicated on the figure. The dashed line represents again the fit to the $L_{1.4 \mathrm{GHz}}$ vs. $L_{40-120}$ correlation, i.e., the equivalent of the IRAS FIR/radio correlation.

\subsection{The warm FIR/radio correlation}

Figure 2 reproduces the relationship between the warm FIR luminosity and $L_{1.4 \mathrm{GHz}}$ for the 72 sample galaxies. The slope of the correlation is $m=1.03 \pm 0.03$ (see Table 3), which indicates that the correlation is linear.

This result differs from the previous one of $\mathrm{Xu}$ et al. (1994b), who derived the warm FIR/radio correlation based on the proportionality between the IRAS FIR and $\mathrm{H} \alpha$ emissions. These authors obtained a non-linear correlation, with the radio-to-FIR luminosity ratio increasing with luminosity. One possible explanation for the non-linearity of the correlation obtained by Xu et al. (1994b) is that the $\mathrm{H} \alpha$ emission was corrected for extinction using the same value for all galaxies. However, if more luminous galaxies suffer from larger amounts of extinction, then the intrinsic $\mathrm{H} \alpha$ emission at the bright end of the correlation was underestimated by $\mathrm{Xu}$ et al., and thus the warm FIR emission was also underestimated, producing the non-linearity of the warm FIR/radio correlation found by these authors.

As for the total FIR/radio correlation, the fact that some galaxies show evidence for mild LINER/Seyfert activity or are in pair/multiple/merging systems does not seem to affect the shape of the correlation (not shown). The slope of the correlation for the 20 galaxies with some activity is $m=0.96 \pm 0.06$ (Table 3). For the remainder we obtain $m=1.06 \pm 0.04$.

The comparison between the warm FIR/radio correlation and the equivalent of the IRAS FIR/radio correlation (plotted with the dashed line in Fig. 2) shows that the shift between the two correlations is much smaller than in the case of the 


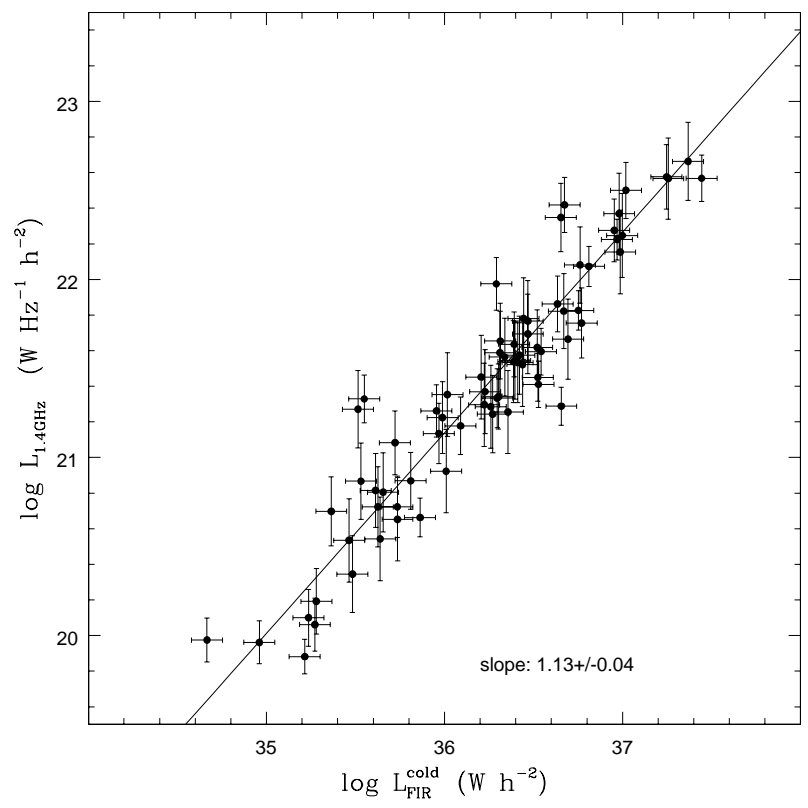

Fig. 3. The radio luminosity at $1.4 \mathrm{GHz}$ vs. the FIR luminosity of the cold component $\left(L_{\mathrm{FIR}}^{\text {cold }}\right)$ for the full sample of 72 objects under investigation. The plot shows a tight correlation between these two emissions, over three orders of magnitude in both luminosities. The fit to the correlation (plotted with solid line) indicates a slightly non-linear correlation. The value of the slope and its uncertainty are indicated on the figure.

total FIR luminosity. In addition, this shift is constant along the luminosity range spanned by the data. This is to be expected, as the IRAS $L_{40-120}$ luminosity is dominated by the contribution of the warm dust component, and contains only a small contribution from the cold dust component.

\subsection{The cold FIR/radio correlation}

Figure 3 reproduces the cold FIR/radio correlation as obtained for the whole sample of 72 objects. We find that the cold FIR/radio correlation is only slightly non-linear $(m=1.13 \pm$ 0.04 , see Table 3 ), similar with what we found for the correlation between $L_{1.4 \mathrm{GHz}}$ and the total FIR luminosity $L_{\mathrm{FIR}}$ (Fig. 1). This is no surprise since the cold dust component dominates the total FIR continuum emission of normal late-type galaxies.

We obtained $m=1.17 \pm 0.08$ (Table 3) for the subsample of 20 galaxies with some activity (LINER/Seyfert or pair/multiple/merging systems) and $m=1.12 \pm 0.05$ for the remainder. Thus, the different mix of galaxies does not seem to play any significant role, as for all correlations presented in this study.

\subsection{The FIR/radio correlation for the Virgo and non-Virgo galaxies}

In their study of cold dust in Virgo Cluster galaxies, Popescu et al. (2002) plotted warm and cold FIR/radio correlations based on the ISO measurements for the 13 galaxies in their sample which had corresponding radio data. Although the statistics were poor, there was an indication that the warm
FIR/radio correlation might be non-linear, whereas in this paper we obtain a linear slope for our sample of 72 objects. Since the sample presented in this work includes the Virgo galaxies from Popescu et al. (2002), we expect to reproduce the same trend when the fit is only applied to the Virgo galaxies. For this we fitted once again the FIR/radio correlations (for the total FIR emission, as well as for the warm and cold FIR emissions), this time separately for the Virgo and non-Virgo galaxies. In total there are 17 Virgo cluster galaxies, comprising the 14 from the VIRGO subsample and 3 Virgo cluster galaxies detected by ISS but not observed by Tuffs et al. (2002a,b). Correspondingly, there are 55 non-Virgo galaxies.

The statistics of the fits are given in Table 3. Indeed, in the case of the warm FIR/radio correlation, there is some indication for a non-linear correlation, while the fit to the non-Virgo sample is linear. Overall, there is a tendency for all correlations to be more non-linear for the Virgo sample than for the non-Virgo sample.

In this context it is appropriate to examine the question of environmental effects. Such influence on the FIR emission was not found for the Virgo galaxies (Popescu et al. 2002; Popescu $\&$ Tuffs 2002b). However, the radio emission may be more susceptible to environmental effects, as the radio emitting medium is less tightly bound to the disk than the FIR emitting medium. One could imagine a scenario in which synchrotron halo electrons are swept away by the ram pressure induced by the relative motion of the galaxies through the intracluster medium. Preferentially, such a removal may be more severe for more quiescent galaxies which have less strongly magnetised halos. This could induce a non-linearity in the FIR/radio correlation. However this should only be applicable to galaxies which are entering the intracluster medium now and therefore we expect only sporadic examples of this phenomenon. We conclude that environmental effects play only a secondary role in shaping the FIR/radio correlation. The main distinction between Virgo and non-Virgo galaxies is rather that the former populate the faint end of the correlation, and are more quiescent.

\section{Interpretation of the warm and cold FIR/radio correlations}

In the previous section we have, for the first time, quantitatively established the correlation between the FIR and radio emission by including the bulk of the FIR luminosity carried by the cold dust component. Furthermore our measurements at three FIR wavelengths allowed a direct determination of the warm and cold FIR emissions. Consequently, separate relations have been derived for the warm and cold FIR/radio relations. In this section we will discuss each of the correlations in terms of the dependence on the star formation rate (SFR).

Detailed mapping observations in the 40-200 $\mu \mathrm{m}$ range of nearby galaxies (e.g. Haas et al. 1998; Hippelein et al. 2003) as well as self-consistent models of the UV/submm SEDs in spiral galaxies (Popescu et al. 2000) are both consistent with the presence of locally heated dust in star-forming complexes and of diffuse dust (Tuffs \& Popescu 2003). The diffuse dust is heated both by non-ionising UV photons and optical 
Table 3. The linear best-fit parameters of the FIR/radio correlation (in log-log).

\begin{tabular}{|c|c|c|c|c|c|}
\hline & & $\begin{array}{c}\text { Whole galaxy } \\
\text { sample } \\
N=72\end{array}$ & $\begin{array}{c}\text { Galaxies with } \\
\text { some activity } \\
N=20\end{array}$ & $\begin{array}{c}\text { Virgo } \\
\text { galaxies } \\
N=17\end{array}$ & $\begin{array}{c}\text { Non-Virgo } \\
\text { galaxies } \\
N=55\end{array}$ \\
\hline \multirow{6}{*}{$\begin{array}{c}L_{1.4 \mathrm{GHz}} \text { vs. } \\
L_{\mathrm{FIR}}\end{array}$} & $m$ & 1.10 & 1.09 & 1.31 & 1.10 \\
\hline & $\epsilon(m)$ & 0.03 & 0.07 & 0.12 & 0.06 \\
\hline & $c$ & -18.53 & -18.35 & -26.29 & -18.68 \\
\hline & $\epsilon(c)$ & 1.23 & 2.52 & 4.23 & 2.07 \\
\hline & $\chi_{\text {red }}^{2}$ & 1.35 & 1.67 & 2.29 & 1.05 \\
\hline & $\sigma$ & 0.13 & 0.14 & 0.15 & 0.12 \\
\hline \multirow{6}{*}{$\begin{array}{c}L_{1.4 \mathrm{GHz}} \text { vs. } \\
L_{\mathrm{FIR}}^{\mathrm{warm}}\end{array}$} & $m$ & 1.03 & 0.96 & 1.27 & 0.98 \\
\hline & $\epsilon(m)$ & 0.03 & 0.06 & 0.12 & 0.05 \\
\hline & $c$ & -15.55 & -12.80 & -23.68 & -13.88 \\
\hline & $\epsilon(c)$ & 1.19 & 2.22 & 4.23 & 1.88 \\
\hline & $\chi_{\text {red }}^{2}$ & 1.15 & 1.26 & 2.15 & 0.82 \\
\hline & $\begin{array}{c}{ }^{2} \text { rea } \\
\sigma\end{array}$ & 0.14 & 0.15 & 0.17 & 0.13 \\
\hline \multirow{6}{*}{$\begin{array}{c}L_{1.4 \mathrm{GHz}} \text { vs. } \\
L_{\mathrm{FIR}}^{\text {cold }}\end{array}$} & $m$ & 1.13 & 1.17 & 1.35 & 1.14 \\
\hline & $\epsilon(m)$ & 0.04 & 0.08 & 0.14 & 0.06 \\
\hline & $c$ & -19.43 & -21.85 & -27.03 & -19.93 \\
\hline & $\epsilon(c)$ & 1.40 & 2.91 & 4.74 & 2.07 \\
\hline & $\chi_{\text {red }}^{2}$ & 1.61 & 2.27 & 2.36 & 1.38 \\
\hline & $\sigma$ & 0.15 & 0.17 & 0.16 & 0.15 \\
\hline \multirow{6}{*}{$\begin{array}{c}L_{1.4 \mathrm{GHz}} \text { vs. } \\
L_{40-120}\end{array}$} & $m$ & 1.04 & 0.93 & 1.29 & 1.01 \\
\hline & $\epsilon(m)$ & 0.03 & 0.06 & 0.10 & 0.05 \\
\hline & $c$ & -16.13 & -12.24 & -25.02 & -15.11 \\
\hline & $\epsilon(c)$ & 1.12 & 2.04 & 3.65 & 1.86 \\
\hline & $\chi_{\text {red }}^{2}$ & 1.28 & 1.61 & 2.85 & 0.75 \\
\hline & $\sigma$ & 0.13 & 0.15 & 0.16 & 0.11 \\
\hline
\end{tabular}

photons (Xu 1990). Then, the relation between SFR and the total FIR energy output can be derived from the following equation:

$L_{\mathrm{FIR}}^{\mathrm{tot}}=L_{\mathrm{FIR}}^{\mathrm{HII}}+L_{\mathrm{FIR}}^{\mathrm{UV}}+L_{\mathrm{FIR}}^{\mathrm{opt}}$

where $L_{\mathrm{FIR}}^{\mathrm{tot}}$ is the total FIR luminosity emitted by the galaxy, $L_{\mathrm{FIR}}^{\mathrm{HII}}$ is the FIR luminosity emitted by the HII regions, $L_{\mathrm{FIR}}^{\mathrm{UV}}$ is the component of the diffuse FIR luminosity powered by the non-ionising UV photons and $L_{\mathrm{FIR}}^{\mathrm{opt}}$ is the component of the diffuse FIR luminosity powered by the optical photons. The equation can be further expressed ${ }^{5}$ in terms of SFR:

$$
\begin{aligned}
L_{\mathrm{FIR}}^{\mathrm{tot}}= & S F R \times\left(L_{0} \times F+L_{x} \times X\right) \\
& +S F R \times L_{0} \times(1-F) \times G_{\mathrm{uv}}+L_{\mathrm{FIR}}^{\mathrm{opt}}
\end{aligned}
$$

where the first term corresponds to $L_{\mathrm{FIR}}^{\mathrm{HII}}$ and the second term to $L_{\mathrm{FIR}}^{\mathrm{UV}}$. SFR - the variable of Eq. (2) - is the present-day star formation rate in $M_{\odot} / \mathrm{yr}, L_{0}$ and $L_{x}$ are the non-ionising and the ionising UV bolometric luminosities of a young stellar population corresponding to $S F R=1 M_{\odot} / \mathrm{yr}$ (which can be derived from population synthesis models), $F$ and $X$ are the fractions of non-ionising and ionising UV emission that are absorbed by dust locally within star forming complexes, and the factor $G_{\mathrm{uv}}$ is the probability that a non-ionising UV photon escaping from the star formation complexes will be absorbed by dust in the diffuse interstellar medium.

\footnotetext{
5 The formulation in Eq. (2) is applicable for the case that the diffuse component is illuminated by a continuous stellar emissivity, and neglects the discrete character of stars.
}

In the way it is defined (see also Popescu et al. 2000), the $F$ factor accounts for the inhomogeneities in the distributions of dust and stars. It determines the additional likelyhood of absorption of non-ionising UV photons due to correlations between an inhomogeneous distribution of young stars and (opaque) parent molecular clouds. In this sense the $F$ factor represents a clumpiness factor in galaxies.

The value of $G_{\mathrm{uv}}$ depends on both the overall amount of diffuse dust and its geometrical distribution relative to the distribution of the young stellar population. The relative distribution of stellar emissivity and dust as a function of SFR is not known. But broadly speaking one would expect SFR to increase with the gas surface density in galaxy disks (Schmidt 1959; Kennicutt 1998). If the dust content is proportional to the gas content ${ }^{6}$, the dust opacity would also increase with SFR (for a fixed geometry). $G_{\mathrm{uv}}$ would then increase with SFR for the optically thin case and would tend asymptotically towards the fixed value of unity for the optically thick case. From this definition it is obvious that the $G_{\mathrm{uv}}$ is a measure of opacity in galaxies.

\footnotetext{
${ }^{6}$ Broadly speaking, the dust content is proportional to the metal abundance (e.g. $[\mathrm{O} / \mathrm{H}]$ ) times the average gas column density of the disk. $[\mathrm{O} / \mathrm{H}]$ is known to increase with the optical luminosity of the galaxy (Zaritsky et al. 1994), enforcing the idea that the metal content of a galaxy like our own is mostly the result of the past star formation activity (e.g. Dwek 1998). In absence of an observationally established relation between metallicity of the disk and SFR, we expect this metallicity to contribute only to the scatter of the relation between dust content and SFR.
} 
The fraction $X$ of ionising UV photons that are absorbed by dust in HII regions exhibits a broad range of values, varying from 0.3-0.7 (Inoue et al. 2001; Inoue 2001). However, even if $X$ approaches unity, the intrinsic luminosity $L_{X}$ is so much smaller than the intrinsic non-ionising UV luminosity $L_{0}$ (Bruzual \& Charlot 1993), that its contribution to $L_{\mathrm{FIR}}^{\mathrm{HII}}$ can still be neglected.

\subsection{The warm FIR/radio correlation}

To interpret the warm FIR/radio correlation, we identify the warm dust component from our fitting procedure with dust locally heated within the HII regions, such that $L_{\mathrm{FIR}}^{\mathrm{warm}} \simeq L_{\mathrm{FIR}}^{\mathrm{HII}}$. In this case $L_{\mathrm{FIR}}^{\mathrm{warm}}$ can be written as:

$L_{\mathrm{FIR}}^{\mathrm{warm}} \simeq S F R \times L_{0} \times F$.

In principle Eq. (3) can be used to constrain the dependence of the radio luminosity on SFR. In Sect. 3.2 we found a linear correlation between the warm FIR and radio luminosities for the sample as a whole. Under the assumption that the clumpiness factor $F$ does not vary with SFR, this would imply a linear relation between radio luminosity and SFR. This is in accordance with the prediction of the calorimeter theory (Völk 1989).

\subsection{The cold FIR/radio correlation}

If the cold dust component is identified with diffuse dust heated by the interstellar radiation field, then:

$L_{\mathrm{FIR}}^{\mathrm{cold}} \simeq S F R \times L_{0} \times(1-F) \times G_{\mathrm{uv}}+L_{\mathrm{FIR}}^{\mathrm{opt}}$.

To interpret Eq. (4) we have to take into account that the opacity $\left(G_{\mathrm{uv}}\right.$ factor) could vary systematically with SFR (see also Bell 2003). As argued above, $G_{\mathrm{uv}}$ should increase with SFR for the optically thin case and should approach unity for the optically thick case. This means that $L_{\mathrm{FIR}}^{\text {cold }}$ would increase more than linearly with increasing SFR for the optically thin case and would tend towards linearity for the extreme optically thick case. In Sect. 3.3 we found a slightly non-linear cold FIR/radio correlation ( $m=1.13$ with a $3 \sigma$ lower limit on the slope of 1.01) for the sample as a whole, in the sense that the ratio radio-to-FIR increases more than linearly with increasing luminosity, the opposite trend than that predicted by opacity. Could this observed trend be explained at low luminosities by considering an increased contribution of the heating of dust by optical photons? Xu et al. (1994a) studied the effect of the optical heating on the FIR/radio correlation and found that this would indeed introduce a non-linearity at low luminosities. However they assumed that $G_{\mathrm{uv}}$ was the same for all galaxies, irrespective of SFR. In fact the opacity should have its strongest dependence on SFR at low luminosities. As argued by Bell (2003), the effect of optical heating is negligible compared with the effect of opacity. Thus, most probably, the optical heating is not enough to explain the observed slope at low luminosities. One possibility to explain the observed slope of the cold FIR/radio correlation is that the radio emission increases more than linearly with increasing SFR for the optically thin case $\left(G_{\mathrm{uv}}<1\right)$ and has a marginal deviation from linearity for the optically thick case $\left(G_{\mathrm{uv}}\right.$ approaches 1$)$. But this is in contradiction with our inference from the warm FIR/radio correlation that the radio luminosity has a linear dependence on SFR.

\section{Discussion}

We have seen in the previous section that it is not easy to identify a scenario which can simultaneously account for both the warm and cold FIR/radio correlations. Whereas the warm FIR/radio correlation was consistent with a linear dependence of the radio on SFR, the cold FIR/radio correlation was more readily interpreted in terms of a non-linear dependence of the radio emission on SFR. To reconcile these seemingly conflicting results we need to re-examine the underlying assumptions on which the results were based.

In drawing the conclusion from the warm FIR/radio correlation we assumed that the clumpiness factor $(F)$ is a constant. However, if an increased SFR is accompanied not only by an increase in the number of independent HII regions, but also by a higher probability for further star formation to happen preferentially near already existing HII regions, then the $F$ factor would also increase, as a consequence of the increased blocking capability of the optically thick molecular clouds in the star-forming complex (e.g. Witt \& Gordon 1996). This would be expected to occur if star formation is a self-propagating phenomenon, in which preceding generations of stars can trigger the formation of new generations.

Referring again to Eq. (3), we see that, if the clumpiness factor $F$ increases with SFR, then the $L_{\mathrm{FIR}}^{\text {warm }}$ increases more rapidly than linearly with SFR. In turn, the radio luminosity, which empirically was found to be proportional to $L_{\mathrm{FIR}}^{\mathrm{warm}}$, will also increase more rapidly than linearly. Qualitatively this is what is needed to bring the warm FIR/radio correlation into consistency with the cold FIR/radio correlation. In Eq. (4) the effect of increasing the clumpiness factor $F$ with SFR would depress $L_{\mathrm{FIR}}^{\text {cold }}$. This, together with the non-linear dependence of the radio on the SFR, would mitigate the trends introduced by opacity, leading to an increased ratio radio/cold FIR with increasing SFR.

From this discussion we conclude that one possibility to bring into consistency the warm and cold FIR/radio correlations would be to invoke a non-linear dependence of the radio emission on SFR. Such a non-linear dependence could be understood in terms of an increase in the residence time of the synchrotron-emitting electrons with increasing galaxy size, as postulated by Chi \& Wolfendale (1990). However, the same trend can also be obtained within the framework of the calorimeter theory, if the radio emission is identified with the sum of diffuse and localised source components. The localised source component is comprised of synchrotron emission from supernova remnants (which account for about 10 per cent of the total radio flux density of galaxies at $1.4 \mathrm{GHz}$ - Lisenfeld \& Völk 2000) plus thermal emission from star forming regions. The diffuse component consists of a pure synchrotron emission component, as given by Völk (1989), plus an additional diffuse free-free component. The free-free component arises from the warm ionised medium, as defined by McKee \& Ostriker (1977), and its emission is in general much smaller 
than the synchrotron emission. Nevertheless we expect that the relative contribution of the synchrotron and free-free components to vary with SFR, such that at high SFR the free-free component becomes non-negligible. This is because the filling factor of the warm ionised medium increases with increasing SFR. Since the non-thermal diffuse radio emission is still proportional with SFR (Völk 1989), then the increased fraction of free-free emission with increasing SFR will introduce the nonlinearity in the radio emission.

In this paper we have used a physical though qualitative description of the warm and cold FIR/radio correlations. To quantitatively model these correlations we need to derive the dependence on SFR of both the FIR and the radio emissions. From the point of view of the FIR emission, we need to selfconsistently analyse the UV to submm SED of each individual galaxy (Popescu et al. 2000; Popescu \& Tuffs 2002a). In this way the variation of the terms in Eqs. (3) and (4) - clumpiness (the $F$ factor) and opacity ( $G$ factor) - with SFR can be derived. From the point of view of the radio emission we need to determine the relative contribution of its components and express them in terms of the SFR. Such a quantitative treatment would throw insights into the physical processes that make normal galaxies the "machines" they are, ranging from particle accelerators and magnetic field dynamos, to radiative coolants.

\section{Summary}

In this paper we present the first analysis of the FIR/radio correlation which incorporates the bulk of the FIR luminosity radiating longwards of the spectral coverage of IRAS. For this we constructed a composite sample of normal galaxies measured with the ISOPHOT instrument on board ISO. The sample was a combination of the ISOPHOT Virgo Cluster Deep Sample (Tuffs et al. 2002a,b) and of the ISOPHOT Serendipity Sample (Stickel et al. 2000). This provided us with a high dynamic range in star formation activity as well as good statistics. All galaxies in the composite sample have had their integrated flux densities measured by ISOPHOT at $170 \mu \mathrm{m}$ and by ISOPHOT (Virgo subsample) or IRAS (Serendipity subsample) at 60 and $100 \mu \mathrm{m}$. The luminosities of the warm and cold dust emission components were extracted from the available measurements at the three FIR wavelengths. This allowed us to define the FIR/radio correlation separately for the warm and cold dust components.

The inclusion of the cold dust component produces a tendency for the total FIR/radio correlation to become non-linear. We found that the cold FIR/radio correlation is slightly nonlinear, whereas the warm FIR/radio correlation is linear. In order to interpret the individual warm and cold correlations in terms of SFR, we identified the warm emission component with dust locally heated within the HII regions, whereas the cold emission component was identified with diffuse dust heated by the interstellar radiation field. Because the effect of opacity in galaxies would introduce a non-linearity in the coldFIR/radio correlation, in the opposite sense to that observed, both the radio and the FIR emissions are likely to have a nonlinear dependence on SFR (for the range of luminosities covered by our sample, which lies below the luminosity range of starburst galaxies). The behaviour of the radio emission can be accounted for by considering the radio emission to be a superposition of a diffuse synchrotron component, a diffuse free-free component and a localised source component.

Acknowledgements. This research has made use of the NASA/IPAC Extragalactic Database (NED), which is operated by the Jet Propulsion Laboratory, California Institute of Technology, under contract with the National Aeronautics and Space Administration.

\section{References}

Alonso-Herrero, A., Rieke, M. J., Rieke, G. H., \& Shields, J. C. 2000, ApJ, 530, 688

Barth, A. J., Ho, L. C., Filippenko, A. V., \& Sargent, W. L. W. 1998, ApJ, 496, 133

Beck, R. \& Golla, G. 1988, A\&A, 191, L9

Bell, E. F. 2003, ApJ, 586, 794

Binggeli, B., Sandage, A., \& Tammann, G. A. 1985, ApJ, 294, 81 (VCC)

Binggeli, B., Popescu, C. C., \& Tammann, G. A. 1993, A\&AS, 98, 275

Boller, T., Brandt, W. N., Fabian, A. C., \& Fink, H. H. 1997, MNRAS, 289,393

Bonatto, C., Bica, E., \& Alloin, D. 1989, A\&A, 226, 23

Bressan, A., Silva, L. \& Granato, G. L. 2002, A\&A, 392, 377

Bruzual, G., \& Charlot, S. 1993, ApJ, 405, 538

Chi, X., \& Wolfendale, W. 1990, MNRAS, 245, 101

Chini, R., Kreysa, E., Krügel, E., \& Mezger, P. G. 1986, A\&A, 166, L8

Condon, J. J. 1992, ARA\&A, 30, 575

Condon, J. J., Anderson, M. L., \& Helou, G. 1991, ApJ, 376, 95

Condon, J. J., Cotton, W. D., Greisen, E. W., et al. 1998, AJ, 115, 1693

Cox, M. J., Eales, S. A. E., Alexander, P., \& Fitt, A. J. 1988, MNRAS, 235,1227

de Jong, T., Clegg, P. E., Soifer, B. T., et al. 1984, ApJ, 278, L67

de Jong, T., Klein, U., Wielebinski, R., \& Wunderlich, E. 1985, A\&A, 147, L6

Devereux, N. A., \& Eales, S. A. 1989, ApJ, 340, 708

Dwek, E. 1998, ApJ, 501, 643

Fitt, A. J., Alexander, P., \& Cox, M. J. 1988, MNRAS, 233, 907

Gavazzi, G., Cocito, A., \& Vettolani, G. 1986, ApJ, 305, L15

Gonzalez-Delgado, R. M., Perez, E., Tadhunter, C., Vilchez, J. M., \& Rodriguez-Espinosa, J. M. 1997, ApJS, 108, 155

Groves, B.A., Cho, J., Dopita, M., \& Lazarian, A. 2003, PASA, 20, 252

Haas, M., Lemke, D., Stickel, M., et al. 1998, A\&A, 338, L33

Helou, G., Soifer, B. T., \& Rowan-Robinson, M. R. 1985, ApJ, 298, L7

Helou, G., Khan, I. R., Malek, L., \& Boehmer, L. 1988, ApJS, 68, 151

Hibbard, J. E., \& van Gorkom, J. H. 1996, AJ, 111, 655

Hippelein, H., Haas, M., Lemke, D., et al. 2003, A\&A, 407, 137

Ho, L. C., Filippenko, A. V., \& Sargent, W. L. W. 1995, ApJS, 98, 477

Ho, L. C., Filippenko, A. V., \& Sargent, W. L. W. 1997, ApJS, 112, 315

Hoernes, P., Berkhuijsen, E. M., \& Xu, C. 1998, A\&A, 334, 57

Inoue, A. K. 2001, AJ, 122, 1788

Inoue, A. K., Hirashita, H., \& Kamaya, H. 2001, ApJ, 555, 613

Keel, W. C. 1983, ApJS, 52, 229

Kennicutt, R. C. 1998, ApJ, 498, 181

Kessler, M. F., Steinz, J. A., Anderegg, M. E., et al. 1996, A\&A, 315, L27 
Leech, K. J., Völk, H. J., Heinrichsen, I., et al. 1999, MNRAS, 310, 317

Lemke, D., Klaas, U., Abolins, J., et al. 1996, A\&A, 315, L64

Lisenfeld, U., \& Völk, H. J. 2000, A\&A, 354, 423

Lisenfeld, U., Völk, H. J., \& Xu, C. 1996a, A\&A, 306, 677

Lisenfeld, U., Völk, H. J., \& Xu, C. 1996b, A\&A, 314, 745

McKee, C. F., \& Ostriker, J. P. 1977, ApJ, 218, 148

Misiriotis, A., Popescu, C. C., Tuffs, R. J., \& Kylafis, N. D. 2001, A\&A, 372, 775

Niklas, S., Klein, U., \& Wielebinski, R. 1995, A\&A, 293, 56

Page, M. J., Mittaz, J. P. D., \& Carrera, F. J. 2001, MNRAS, 325, 575

Peeters, E., Martín-Hernández, N. L., Damour, F., et al. 2002, A\&A, 381,571

Pierini, D., Leech, K. J., Tuffs, R. J., \& Völk, H. J. 1999, MNRAS, 303, 29.

Pierini, D., Lequeux, J., Boselli, A., Leech, K. J., \& Völk, H. J. 2001, A\&A, 373, 827

Pierini, D., Leech, K. J., \& Völk, H. J. 2003, A\&A, 397, 871

Popescu, C. C., \& Tuffs, R. J. 2002a, in Five Days of Creation: Astronomy with Large Telescopes from Ground and Space, Reviews in Modern Astronomy, vol. 15, ed. R. E. Schielicke (Hamburg: Astronomische Gesellschaft), 239

Popescu, C. C., \& Tuffs, R. J. 2002b, MNRAS, 335, L41

Popescu, C. C., Misiriotis, A., Kylafis, N. D., Tuffs, R. J., \& Fischera, J. 2000, A\&A, 362, 138

Popescu, C. C., Tuffs, R. J., Völk, H. J., Pierini, D., \& Madore, B. F. 2002, ApJ, 567, 221

Press, W. H., Flannery, B. P., Teukolsky, S. A., \& Vetterling, W. T. 1992, Numerical Recipes, 2nd ed. (Cambridge: University Press)

Price, R., \& Duric, N. 1992, ApJ, 401, 81

Rauscher, B. J. 1995, AJ, 109, 1608

Risaliti, G., Gilli, R., Maiolino, R., \& Salvati, M. 2000, A\&A, 357, 13
Rodriguez Espinosa, J. M., Rudy, R. J., \& Jones, B. 1987, ApJ, 312, 555

Schmidt, M. 1959, ApJ, 129, 243

Sharples, R. M., Longmore, A. J., Hawarden, T. G., \& Carter, D. 1984, MNRAS, 208, 15

Silva, L., Granato, G. L., Bressan, A., \& Danese, L. 1998, ApJ, 509, 103

Smith, D. A., Herter, T., Haynes, M. P., Beichman, C. A., \& Gautier, T. N. 1996, ApJS, 104, 217

Sopp, H. M., \& Alexander, P. 1991, MNRAS, 251, 14

Stauffer, J. R. 1982, ApJ, 262, 66

Stickel, M., Lemke, D., Klaas, U., et al. 2000, A\&A, 359, 865

Tuffs, R. J., \& Popescu, C. C. 2003, in Exploiting the ISO Data Archive. Infrared Astronomy in the Internet Age, ed. C. Gry et al., ESA Publications Series, ESA SP-511, 239

Tuffs, R. J., Popescu, C. C., Pierini, D., et al. 2002a, ApJS, 139, 37

Tuffs, R. J., Popescu, C. C., Pierini, D., et al. 2002b, ApJS, 140, 609

Tully, R. B., \& Shaya, E. J. 1984, ApJ, 281, 31

Völk, H. J. 1989, A\&A, 218, 67

Völk, H. J., \& Xu, C. 1994, Infrared Physics and Technology, 35, 527

Vorontsov-Yelyaminov, B., \& Arhipova, V. P. 1968, Morphological Catalogue of Galaxies, vol. 4 (Moscow: Moscow State University)

Witt, A. N., \& Gordon, K. D. 1996, ApJ, 463, 681

Wunderlich, E., \& Klein, U. 1991, A\&AS, 87, 247

Wunderlich, E., Wielebinski, R., \& Klein, U. 1987, A\&AS, 69, 487

Xu, C. 1990, ApJ, 365, L47 A\&A, 257, 47

Xu, C., Lisenfeld, U., Völk, H. J., \& Wunderlich, E. 1994a, A\&A, 282, 19

Xu, C., Lisenfeld, U., \& Völk, H. J. 1994b, A\&A, 285, 19

Zaritsky, D., Kennicutt, R. C., \& Huchra, J. P. 1994, ApJ, 420, 87 
D. Pierini et al.: The far-infrared/radio correlation in the ISO era, Online Material $p 1$

\section{Online Material}




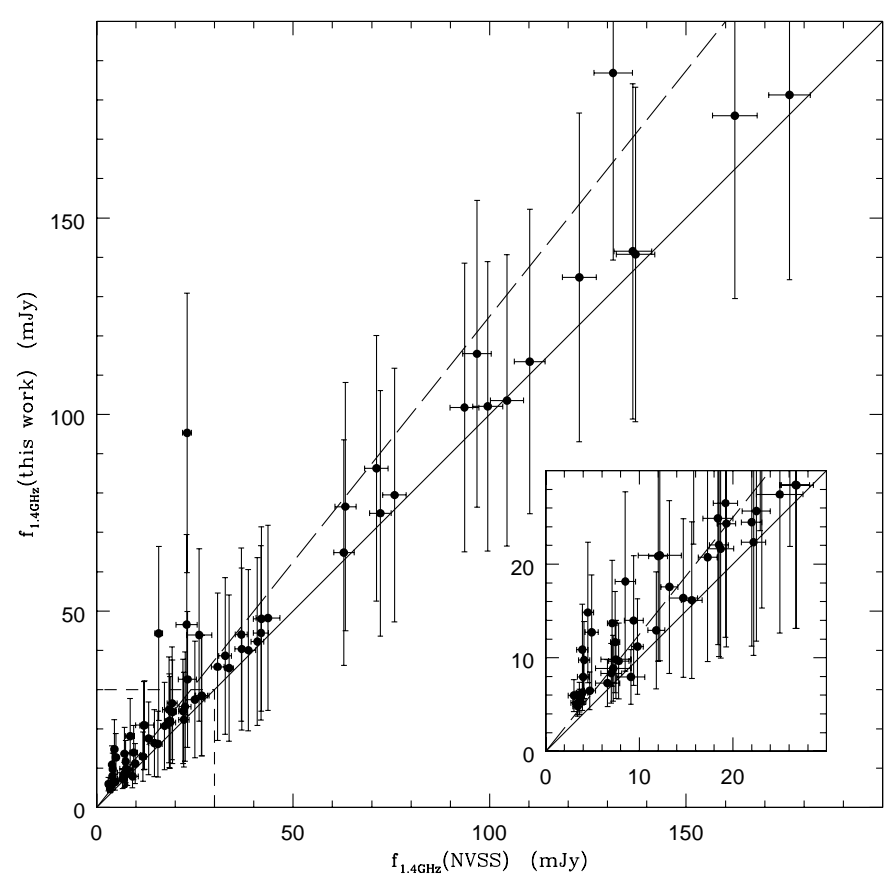

Fig. A.1. Comparison of the radio fluxes at $1.4 \mathrm{GHz}$ determined in this study from direct aperture photometry of the NVSS images and the NVSS catalogue fluxes for the radio counterparts of the 72 sample objects under investigation. The latter fluxes are obtained from Gaussian model components fitted to the survey images. Here the solid and long-dashed lines represent the 1:1 and 1.25:1 ratios, respectively, while the box delimited by a short-dashed line corresponds to the region zoomed in the enclosed box. On average, our fluxes are 25 per cent larger than the NVSS catalogue ones (see text).

\section{Appendix A: The extraction of the radio flux densities from the NVSS maps}

The NVSS used the NRAO Very Large Array telescope and covers the sky north of a declination of -40 degrees at a frequency of $1.4 \mathrm{GHz}$, a resolution of $45^{\prime \prime}$ and a limiting peak source brightness of about $2.5 \mathrm{mJy}^{\text {beam }}{ }^{-1}$ (Condon et al. 1998). Radio counterparts are associated with optically catalogued galaxies when the distance of the peak radio surface brightness of the potential radio identification from the optical position of the target galaxy is within the NVSS resolution. The probability of finding an NVSS source within $45^{\prime \prime}$ of an arbitrary position is 0.02 .

As a result of this survey a source catalogue has been produced, which consists of Gaussian model components fitted to the survey images. If the source is significantly larger than the resolution of the survey then it may be represented by several of these components. The fitted parameters are deconvolved from the instrumental resolution producing source sizes (or upper limits with 99 per cent confidence) and the integrated flux density of the model. Error estimates for each of the source parameters are also given.

In addition to the catalogue, a postage stamp server which returns FITS files of limited regions from the NVSS calibrated images is available electronically. The noise level in these images is about $0.5 \mathrm{mJy}$ beam $^{-1}$. We have decided to extract our own aperture photometry from these calibrated maps under the consideration that some of our sample galaxies might have failed the classification criteria of the NVSS catalogue and/or not be represented by Gaussian model components at best and/or have unaccounted faint extended emission. This question is crucial, since the $45^{\prime \prime}$ beam of the NVSS has a much smaller aperture than the ISOPHOT beam at $170 \mu \mathrm{m}\left(92^{\prime \prime}\right)$. Aperture photometry was extracted with the task QPHOT in IRAF $^{7}$.

We plot the comparison between our photometry ( $y$ axis) and the NVSS catalogue one ( $x$ axis) in Fig. A.1. Here the solid and long-dashed lines represent the 1:1 and 1.25:1 ratios, respectively, while the box delimited by a short-dashed line corresponds to the region zoomed in the enclosed box. As expected, we find that the agreement is excellent for those radio sources which appear unresolved with the NVSS beam but it becomes worse in case of very extended/complex sources. On average, our fluxes are 25 per cent larger than the NVSS catalogue ones.

\section{Appendix B: Photometric comparison between the ISOPHOT Serendipity Survey and the ISOPHOT Deep Virgo Cluster Survey at $\mathbf{1 7 0} \mu \mathrm{m}$}

Four galaxies from the ISOPHOT Virgo Cluster Deep Survey (Tuffs et al. 2002a,b) were also detected by the ISOPHOT $170 \mu \mathrm{m}$ Serendipity Survey (Stickel et al. 2000). These galaxies are: VCC 66, 460, 836 and 873, alias NGC 4178, 4293, 4388 and 4402, respectively. From the comparison of the $170 \mu \mathrm{m}$ flux densities of these 4 galaxies it emerges that the values of Stickel et al. are systematically higher than those of Tuffs et al., the difference being significant (a factor of 2) for VCC 836 and 873.

The absolute calibration is not a reason for this, since background measurements averaged over the 4 common objects are the same to within a few per cent. However the different techniques used to derived photometry may give rise to differences. Even for extended sources, the Serendipity Survey photometry is extracted by fitting circular gaussians to the strip maps, whereas the Virgo sample photometry is performed by fitting elliptical gaussians. Thus, one may expect the Serendipity Survey $170 \mu \mathrm{m}$ fluxes to be overestimated as inclination gets larger, as indeed it is the case for the previous 4 galaxies. In addition, in the Serendipity Survey photometry there is a spread of 30 per cent for bright sources, due to systematic effects like offset of source from scan line. To this must be added random errors for faint sources (10 Jy or less). The combined uncertainties can be 50 per cent for faint sources (see Fig. 2 of Stickel et al. 2000). Finally, compared with Tuffs et al. (2002a,b), Stickel et al. made larger corrections for the loss of signal incurred by the transient response of the detector to illumination steps - the so-called "transient correction". The multiplicative corrections of Stickel et al. range from 1.1 for a faint source to a factor of 2 for sources of $60 \mathrm{Jy}$. By comparison the transient

\footnotetext{
${ }^{7}$ IRAF is the Image Analysis and Reduction Facility made available to the astronomical community by the National Optical Astronomy Observatories, which are operated by AURA, Inc., under contract with the US National Science Foundation.
} 
D. Pierini et al.: The far-infrared/radio correlation in the ISO era, Online Material p 3

Table 2. Galaxy parameters for the ISS subsample.

\begin{tabular}{|c|c|c|c|c|c|c|c|c|}
\hline $\begin{array}{l}\text { Den. } \\
\text { NGC/UGC or other }\end{array}$ & Hubble type & $\begin{array}{c}\log L_{1.4 \mathrm{GHz}} \\
{\left[\mathrm{W} \mathrm{Hz}^{-1}\right]}\end{array}$ & $\begin{array}{l}\log L_{\mathrm{FIR}} \\
{\left[\mathrm{W} \mathrm{h}^{-2}\right]}\end{array}$ & $\begin{array}{c}\log L_{\mathrm{FIR}}^{\mathrm{Warm}} \\
{\left[\mathrm{W} \mathrm{h}^{-2}\right]}\end{array}$ & $\begin{array}{l}\log L_{\mathrm{FIR}}^{\text {cold }} \\
{\left[\mathrm{W} \mathrm{h}^{-2}\right]}\end{array}$ & $\begin{array}{c}\log L_{40-120} \\
{\left[\mathrm{~W} \mathrm{~h}^{-2}\right]}\end{array}$ & $\begin{array}{c}\text { Dist. } \\
{\left[\mathrm{Mpc} \mathrm{h}^{-1}\right]}\end{array}$ & Notes \\
\hline NGC 7821 & Scd pec sp & $22.37 \pm 0.23$ & 37.19 & 36.77 & 36.98 & 36.99 & 73.39 & \\
\hline NGC 157 & $\mathrm{SAB}(\mathrm{rs}) \mathrm{bc}$ & $21.83 \pm 0.11$ & 36.88 & 36.28 & 36.75 & 36.47 & 16.68 & \\
\hline MCG -05-03-020 & $\mathrm{SAB}(\mathrm{r}) \mathrm{c}$ : & $21.29 \pm 0.11$ & 36.73 & 35.91 & 36.66 & 36.26 & 56.09 & AGN? $?^{a, b}$ \\
\hline UGC 816 & $\mathrm{Sc}$ & $22.35 \pm 0.19$ & 36.90 & 36.53 & 36.65 & 36.72 & 51.88 & \\
\hline NGC 477 & $\mathrm{SAB}(\mathrm{s}) \mathrm{c}$ & $21.45 \pm 0.13$ & 36.72 & 36.28 & 36.52 & 36.42 & 58.76 & \\
\hline NGC 520 & & $22.07 \pm 0.11$ & 37.17 & 36.93 & 36.81 & 36.97 & 23.37 & GPair? $^{\mathrm{c}}$ \\
\hline NGC 549 & $\left(\mathrm{R}^{\prime}\right) \mathrm{SB}(\mathrm{s}) 0 / \mathrm{a}$ & $21.64 \pm 0.18$ & 36.53 & 35.98 & 36.39 & 36.16 & 61.76 & \\
\hline MCG -05-05-007 & $\mathrm{Sbc}$ & $21.41 \pm 0.13$ & 36.61 & 35.84 & 36.53 & 36.09 & 59.04 & \\
\hline UGC 1560 & $\left(\mathrm{R}^{\prime}\right) \mathrm{SB}(\mathrm{s}) \mathrm{b}$ : & $22.08 \pm 0.21$ & 37.00 & 36.64 & 36.76 & 36.83 & 84.91 & \\
\hline UGC 2238 & Im? & $22.58 \pm 0.18$ & 37.46 & 37.05 & 37.25 & 37.30 & 64.36 & LINER $^{\mathrm{d}}$ \\
\hline NGC 1087 & $\mathrm{SAB}(\mathrm{rs}) \mathrm{c}$ & $21.60 \pm 0.13$ & 36.69 & 36.15 & 36.54 & 36.26 & 15.19 & \\
\hline MCG -03-12-002 & & $22.66 \pm 0.22$ & 37.53 & 37.03 & 37.37 & 37.45 & 95.42 & GPair $^{\mathrm{e}}$ \\
\hline UGC 3066 & $\mathrm{SAB}(\mathrm{r}) \mathrm{d}$ & $21.57 \pm 0.22$ & 36.55 & 35.95 & 36.42 & 36.20 & 46.39 & \\
\hline NGC 1614 & $\mathrm{SB}(\mathrm{s}) \mathrm{c}$ pec & $22.57 \pm 0.13$ & 37.59 & 37.03 & 37.44 & 37.55 & 47.78 & $\mathrm{Sy} 2^{\mathrm{f}, \mathrm{g}}$ \\
\hline NGC 1667 & $\mathrm{SAB}(\mathrm{r}) \mathrm{c}$ & $22.28 \pm 0.18$ & 37.15 & 36.73 & 36.95 & 36.90 & 45.46 & Sy $2^{\mathrm{h}}$ \\
\hline MCG-03-13-051 & $\left(\mathrm{R}^{\prime}\right) \mathrm{SB}(\mathrm{s}) \mathrm{b}$ & $21.75 \pm 0.20$ & 36.89 & 36.28 & 36.77 & 36.49 & 66.40 & \\
\hline NGC 2958 & S(r)bc & $21.82 \pm 0.21$ & 36.85 & 36.39 & 36.67 & 36.56 & 66.63 & \\
\hline NGC 3183 & $\mathrm{SB}(\mathrm{s}) \mathrm{bc}$ & $21.69 \pm 0.22$ & 36.62 & 36.09 & 36.47 & 36.41 & 30.88 & \\
\hline MCG-06-23-029 & Sbc : sp & $21.35 \pm 0.23$ & 36.26 & 35.90 & 36.02 & 36.06 & 31.22 & \\
\hline NGC 4222 & $\mathrm{Sc}$ & $19.88 \pm 0.10$ & 35.35 & 34.77 & 35.21 & 35.00 & 11.50 & \\
\hline NGC 4383 & Sa? pec & $20.80 \pm 0.22$ & 35.99 & 35.72 & 35.65 & 35.77 & 11.50 & \\
\hline NGC 4639 & $\mathrm{SAB}(\mathrm{rs}) \mathrm{bc}$ & $20.19 \pm 0.18$ & 35.42 & 34.85 & 35.28 & 35.16 & 11.50 & Sy $1.8^{\mathrm{h}}$ \\
\hline MCG -05-31-035 & $\mathrm{SB}(\mathrm{rs}) \mathrm{d}$ & $20.81 \pm 0.21$ & 35.81 & 35.37 & 35.61 & 35.54 & 22.16 & \\
\hline NGC 4981 & $\mathrm{SAB}(\mathrm{r}) \mathrm{bc}$ & $20.92 \pm 0.23$ & 36.15 & 35.59 & 36.01 & 35.83 & 16.86 & LINER $^{\mathrm{i}}$ \\
\hline MCG -05-31-039 & $\mathrm{SB}(\mathrm{s}) \mathrm{d}: \mathrm{sp}$ & $20.72 \pm 0.17$ & 35.89 & 35.38 & 35.73 & 35.58 & 23.81 & \\
\hline IC 4221 & SA(r)c pec? & $20.87 \pm 0.16$ & 35.98 & 35.50 & 35.81 & 35.70 & 28.95 & \\
\hline NGC 5085 & $\mathrm{SA}(\mathrm{s}) \mathrm{c}$ & $21.24 \pm 0.22$ & 36.38 & 35.71 & 36.27 & 35.98 & 19.56 & \\
\hline NGC 5292 & $\left(\mathrm{R}^{\prime}\right) \mathrm{SA}(\mathrm{rs}) \mathrm{ab}$ & $21.34 \pm 0.18$ & 36.41 & 35.75 & 36.30 & 36.05 & 44.66 & \\
\hline UGC 8739 & SB? & $22.50 \pm 0.16$ & 37.25 & 36.86 & 37.02 & 37.02 & 50.95 & \\
\hline MCG -04-33-013 & Sa : pec sp & $21.52 \pm 0.23$ & 36.55 & 35.91 & 36.44 & 36.19 & 34.55 & \\
\hline NGC 5468 & $\mathrm{SAB}(\mathrm{rs}) \mathrm{cd}$ & $21.65 \pm 0.21$ & 36.52 & 36.11 & 36.31 & 36.32 & 28.45 & \\
\hline NGC 5504 & $\mathrm{SAB}(\mathrm{s}) \mathrm{bc}$ & $21.77 \pm 0.23$ & 36.65 & 36.19 & 36.47 & 36.40 & 52.47 & \\
\hline NGC 5533 & $\mathrm{SA}(\mathrm{rs}) \mathrm{ab}$ & $21.59 \pm 0.23$ & 36.39 & 35.62 & 36.31 & 36.12 & 38.66 & \\
\hline NGC 5604 & Sa pec? & $21.37 \pm 0.23$ & 36.41 & 35.96 & 36.23 & 36.15 & 27.48 & \\
\hline UGC 9483 & $\mathrm{~S}$ & $20.72 \pm 0.22$ & 35.79 & 35.28 & 35.63 & 35.53 & 16.36 & \\
\hline NGC 5757 & $\left(\mathrm{R}^{\prime}\right) \mathrm{SB}(\mathrm{r}) \mathrm{b}$ & $21.54 \pm 0.22$ & 36.65 & 36.29 & 36.40 & 36.38 & 26.30 & \\
\hline NGC 5899 & $\mathrm{SAB}(\mathrm{rs}) \mathrm{c}$ & $21.62 \pm 0.21$ & 36.65 & 36.06 & 36.52 & 36.27 & 25.62 & $\mathrm{Sy} 2^{\mathrm{j}}$ \\
\hline NGC 5956 & Scd? & $20.70 \pm 0.19$ & 35.47 & 34.80 & 35.36 & 35.24 & 18.98 & \\
\hline NGC 5961 & S? & $21.27 \pm 0.22$ & 35.74 & 35.35 & 35.51 & 35.51 & 17.66 & \\
\hline NGC 5985 & $\mathrm{SAB}(\mathrm{r}) \mathrm{b}$ & $21.25 \pm 0.23$ & 36.41 & 35.48 & 36.36 & 35.93 & 25.17 & LINER, Sy $1^{\mathrm{k}}$ \\
\hline NGC 6052 & & $22.42 \pm 0.15$ & 37.04 & 36.79 & 36.67 & 36.87 & 44.99 & GPair $^{\mathrm{d}}$ \\
\hline UGC 10331 & S pec & $21.33 \pm 0.16$ & 36.51 & 36.10 & 36.30 & 36.22 & 44.71 & \\
\hline NGC 6120 & pec & $22.57 \pm 0.23$ & 37.47 & 37.06 & 37.26 & 37.33 & 91.70 & \\
\hline UGC 10524 & $\left(\mathrm{R}^{\prime}\right) \mathrm{SB}(\mathrm{s}) \mathrm{ab}$ & $22.25 \pm 0.23$ & 37.23 & 36.85 & 37.00 & 37.11 & 75.98 & \\
\hline NGC 6381 & $\mathrm{SA}(\mathrm{s}) \mathrm{c}$ ? & $21.22 \pm 0.20$ & 36.20 & 35.80 & 35.99 & 35.98 & 32.65 & \\
\hline NGC 6478 & SAc: & $22.15 \pm 0.23$ & 37.10 & 36.48 & 36.99 & 36.75 & 67.75 & \\
\hline NGC 6574 & $\mathrm{SAB}(\mathrm{rs}) \mathrm{bc}$ : & $21.86 \pm 0.16$ & 36.89 & 36.55 & 36.63 & 36.70 & 22.52 & Sy ${ }^{\mathrm{h}}$ \\
\hline NGC 6585 & $\mathrm{~S} ?$ & $21.45 \pm 0.23$ & 36.39 & 35.93 & 36.21 & 36.15 & 26.35 & \\
\hline NGC 6667 & SABab? pec & $21.28 \pm 0.23$ & 36.42 & 35.91 & 36.26 & 36.22 & 25.82 & \\
\hline NGC 6764 & $\mathrm{SB}(\mathrm{s}) \mathrm{bc}$ & $21.97 \pm 0.15$ & 36.60 & 36.32 & 36.29 & 36.42 & 24.16 & LINER, Sy $2^{1}$ \\
\hline NGC 6796 & Sbc: $\mathrm{sp}$ & $21.30 \pm 0.23$ & 36.36 & 35.81 & 36.22 & 36.20 & 21.89 & \\
\hline NGC 6911 & $\mathrm{SBb}:$ & $21.53 \pm 0.22$ & 36.60 & 36.14 & 36.41 & 36.28 & 25.01 & \\
\hline NGC 6928 & $\mathrm{SB}(\mathrm{s}) \mathrm{ab}$ & $21.66 \pm 0.22$ & 36.83 & 36.26 & 36.69 & 36.52 & 47.07 & \\
\hline NGC 7042 & $\mathrm{Sb}$ & $21.78 \pm 0.23$ & 36.57 & 35.97 & 36.44 & 36.30 & 50.82 & \\
\hline NGC 7172 & Sa pec sp & $21.56 \pm 0.22$ & 36.59 & 36.24 & 36.34 & 36.40 & 26.03 & $\mathrm{Sy} 2^{\mathrm{m}}$ \\
\hline IC 1438 & $\left(\mathrm{R}_{1} \mathrm{R}_{2}^{\prime}\right) \mathrm{SAB}(\mathrm{r}) \mathrm{a}$ & $20.66 \pm 0.11$ & 36.00 & 35.42 & 35.86 & 35.65 & 26.16 & \\
\hline NGC 7541 & $\mathrm{SB}(\mathrm{rs}) \mathrm{bc}$ pec : & $22.22 \pm 0.11$ & 37.22 & 36.86 & 36.97 & 36.98 & 26.78 & \\
\hline NGC 7755 & $\mathrm{SB}(\mathrm{r}) \mathrm{bc}$ & $21.54 \pm 0.23$ & 36.53 & 35.99 & 36.39 & 36.26 & 29.63 & \\
\hline
\end{tabular}

a $\quad$ Boller et al. (1997); ${ }^{\mathrm{b}}$ Page et al. (2001); ${ }^{\mathrm{c}}$ Hibbard \& van Gorkom (1996); ${ }^{\mathrm{d}}$ Smith et al. (1996); ${ }^{\mathrm{e}}$ Vorontsov-Velyaminov \& Arhipova (1968); ${ }^{\mathrm{f}}$ Rodriguez Espinosa et al. (1987);

${ }^{\mathrm{g}}$ Risaliti et al. (2000); ${ }^{\mathrm{h}}$ Gonzalez-Delgado et al. (1997); ${ }^{\mathrm{i}}$ Bonatto et al. (1989); ${ }^{\mathrm{j}}$ Stauffer (1982); ${ }^{\mathrm{k}}$ Ho et al. (1995); ${ }^{1}$ Alonso-Herrero et al. (2000); ${ }^{\mathrm{m}}$ Sharples et al. (1984).

corrections of Tuffs et al. are 10 to 20 per cent only for the C200 detector.

From this discussion we conclude that the Serendipity Survey photometry may be overestimated by a factor of 2 for large (i.e., with apparent major axis longer than $\sim 4^{\prime}$ ) galaxies with inclinations higher than $\sim 70^{\circ}$. 